\title{
Cardiac surgery in the elderly: What goals of care?
}

\author{
Alessandra Pratesi', Francesco Orso', Camilla Ghiara1, Aldo Lo Forte1, Anna Chiara Baroncini', \\ Maria Laura Di Meo', Emanuele Carassi', Samuele Baldasseroni1,2
}

\author{
1 Intensive Geriatric Care Unit, Department of Clinical and Experimental Medicine, University of Florence \\ 2 Division of Cardiology, Department of Cardiovascular and Thoracic Medicine and Surgery, Careggi Hospital, \\ Florence, Italy
}

\begin{abstract}
At present, the majority of cardiac surgery interventions have been performed in the elderly with successful short-term mortality and morbidity; however significant difficulties must be underlined about our capacity to predict long-term outcomes such as disability, worsening quality of life and loss of functional capacity.

The reason probably resides on inability to capture preoperative frailty phenotype with current cardiac surgery risk scores and consequently we are unable to outline the postoperative trajectory of an important patients' centered outcome such as disability free survival. In this perspective, more than one geriatric statement have stressed the systematic underuse of patient reported outcomes in cardiovascular trials even after taking account of their relevance to older feel and wishes. Thus, in the next future is mandatory for geriatric cardiology community closes this gap of evidences through planning of trials in which patients' centered outcomes are considered as primary goals of therapies as well as cardiovascular ones.
\end{abstract}

\section{The current evidences of cardiac surgery in the elderly}

At this time, more than half of cardiac surgery interventions is being performed in patients older than 75 years and this group of patients is steadily rising over time [1].

Corresponding author: Samuele Baldasseroni, Division of Cardiology, Department of Cardiovascular and Thoracic Medicine and Surgery, Careggi Hospital, Firenze, Italy. Tel. +39.055.7949429, 055.7949577.

E-mail: pesine@libero.it

Key words: Cardiac surgery; elderly; outcomes; frailty; disability.

Received for publication: 26 April 2017

Accepted for publication: 4 May 2017

(C) Copyright A. Pratesi et al., 2017

Tipografia PI-ME Editrice, Italy

Monaldi Archives for Chest Disease 2017; 87:852

doi: 10.4081/monaldi.2017.852

This article is distributed under the terms of the Creative Commons Attribution Noncommercial License (by-nc 4.0) which permits any noncommercial use, distribution, and reproduction in any medium. provided the original author(s) and source are credited.
This epidemiological phenomenon has compelled cardiac surgeons to face with a different and more complex clinical scenario represented by patients often accompanied by a larger burden of non-cardiac comorbid conditions and greater illness severity [1]. Nowadays, in the setting of cardiac surgery, elderly patients are more likely to have extensive coronary artery disease and concomitant valvular disease, requiring combined cardiac intervention and need urgent or emergent surgery [2]; nevertheless, new surgery and anesthesiological techniques have resulted in sizeable benefits also for the elderly. However, the clinical and functional complexities of older patient candidate to cardiac surgery have highlighted the significant limitations regarding postoperative predictive power of current cardiac surgery risk scores such as EUROSCORE logistic I and II and STS score [3].

Recently authors showed that the predictive value of many currently available risk-scoring algorithms (ACEF, EUROSCORE I and II, STS score) was insufficient to allow a precise and reliable risk assessment in patients undergoing surgical aortic valve replacement or transcather aortic valve implantation with an overestimation of risk using ACEF and conversely an overestimation of it using EUROSCORE or STS score [3].

The limitations of these risk models reside on their conceptualization and structure, mainly focused on cardiac specific parameters, and consequently in their inability to capture biological and functional vulnerability present in elderly patient and summarized by FRAILTY concept [4].

\section{The role of preoperative frailty}

Frailty is a geriatric clinical domain conceptualized over 3 decades ago as a syndrome characterized by loss of homeostatic reserves and diminished capacity to withstand stress [4]; in clinical practice two conceptual methods of measuring are still operating. A) frailty as a physical phenotype according to the five Fried's criteria [5] - low grip strength, self-reported low energy, slowed walking speed, self-reported low physical activity, and unintentional weight loss. The presence of 3 criteria permit the diagnosis of frailty whereas fulfilling only 1 or 2 of the criteria defines the presence of "pre-frail" condition. B) The Canadian Rockwood approach defines frailty as accumulation deficits across 70 -item related to physical and cognitive state, psychosocial function, and laboratory values; the presence or absence of each item would be classified as a deficit, all deficits are summarized in the 70-item Frailty Index (FI) [6].

Independently from different methods of measure, the presence of frailty is often recognized in older patients candidate to urgent or elective cardiac surgery; data from Canadian registry of cardiac surgery demonstrated that the rate of patients defined as frail increase from $5 \%$ to $10 \%$ in a period from 2005 to 2010 and in patients over 80 years old the rate raised over $20 \%$ [2]. 
Preoperative frailty showed to predict short and long term mortality, morbidity and MACCE in cardiac surgery, furthermore it is able to affect the risk of discharge to nursing home [7]. A potent single domain of physical frailty such as gait speed recently demonstrated in a cohort of 15171 patients with mean age of 71 to capture independently the operative risk with each $0.1-\mathrm{m} / \mathrm{s}$ decrease in gait speed conferring an $11 \%$ increase in mortality [8]. The adverse risk associated with low gait speed $(<0.83 \mathrm{~m} / \mathrm{sec})$ was present independently from type of cardiac intervention and for secondary end-point (in-hospital all-cause death, stroke, prolonged mechanical ventilation, acute kidney injury, deep sternal wound infection, or need for reoperation). Interestingly the authors underlined as using gait speed as the single criterion for frailty definition added a modest predictivity to STS score, suggesting that gait speed measure must be a part of a multi-domain frailty assessment including other geriatric domains such as cognitive, mood evaluation, social and familial network analysis and preoperative health related quality of life measure.

Finally it is crucial to point out that frailty is a dynamic process sometimes with "a priori" unpredictable trajectory after therapeutic interventions; in this perspective Freiheit et al. [9] recently demonstrated that frailty followed a U-shaped curve after revascularization with a temporary improvement in younger but not in older patients (aged $\geq 75$ years) after CABG; in fact these patients showed a continuous increase in their frailty level from baseline during the 30 months of follow-up. Whether this first finding was confirmed in future studies, this different frailty trajectory could be used to inform the patient and individualize the decision-making process about initial treatment choices and allow more tailored subsequent patient care.

\section{The patient centered outcomes: the role of functional capacity, quality of life and disability free-survival}

In all patients candidate to cardiac surgery the major goals are both to alleviate patient symptoms and to improve survival. Indeed, in the surgeon perspective the role of prolonging survival seems overwhelms the patient's wish of providing symptoms' relief and maintaining a good quality of live. The abovementioned dichotomy is significantly emphasized in elderly patients in which the consciousness of being at the end of life makes him oriented towards quality rather than quantity of life [10] (Figure 1). A recent statement of AHA/ACC/AGS underlined the need to close this gap of knowledge in older population with cardiovascular diseases, incorporating patients' goals of care before major cardiac interventions, with the aim to orient choice of therapy and the decision-making process towards preferences and wishes of older patients themselves [11].

Traditionally, in the field of cardiac surgery trials and registries, we divide outcome measures in short and long term ones, systematically including death and serious complications such as acute MI and stroke often summarized in MACCE composite endpoint.

Recovery time such as ICU length to stay, duration of ventilation is often registered after cardiac surgery; these outcomes in the elderly are significantly influenced by the onset of delirium, a serious and frequent complication after cardiac surgery in the elderly population. In this population perioperative delirium is associated with an increased

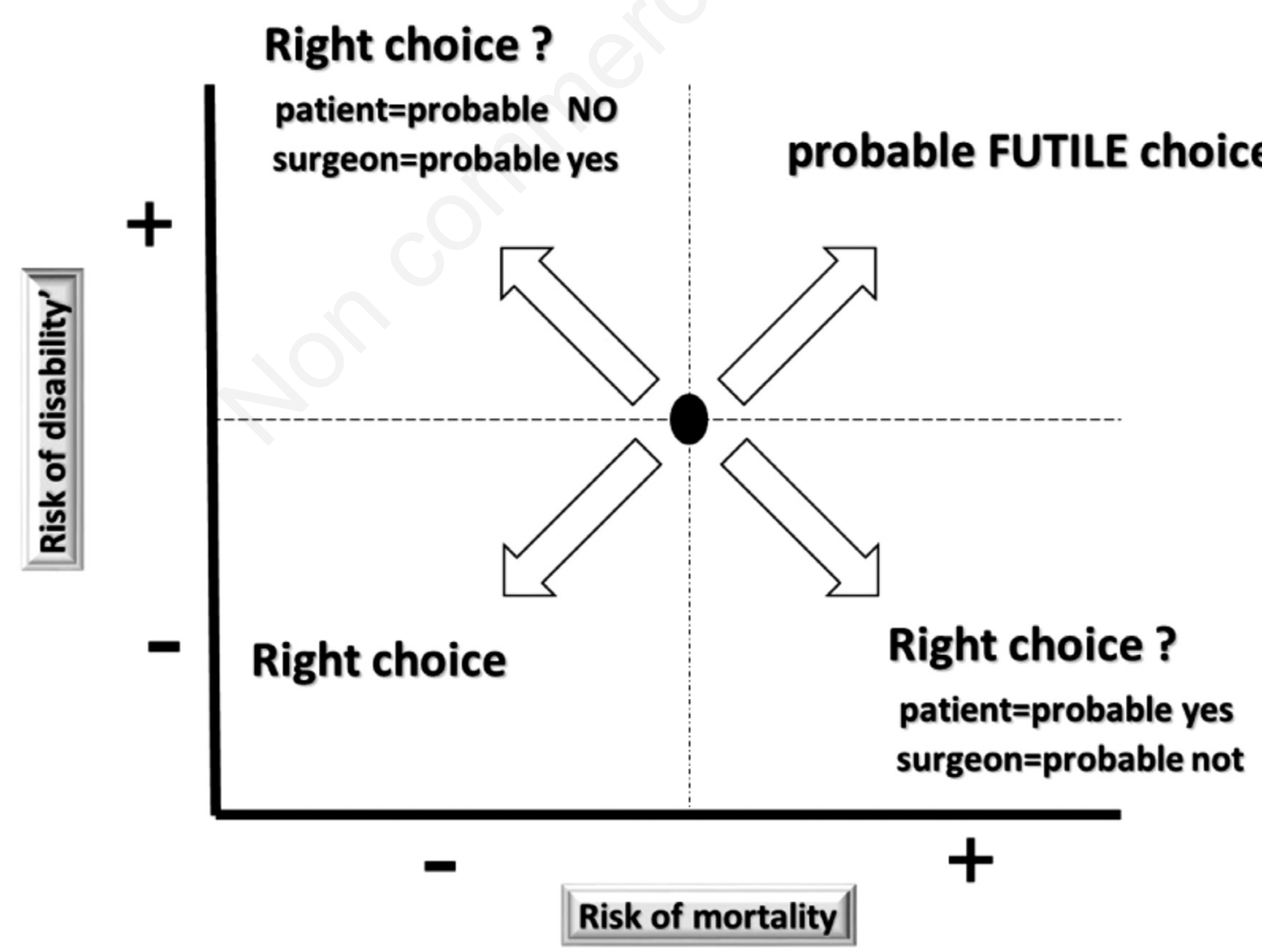

Figure 1. Clinical scenario after cardiac surgery in the elderly: surgeon or patient perspective. 
risk of death, institutionalization, and possibly postoperative overt dementia and it showed to be independently associated with the presence of preoperative frailty.

In this context, recently The Neurologic Academic Research Consortium [12] proposed standardized neurological endpoints for cardiovascular clinical trials, encouraging investigators to incorporate standard assessment of perioperative cognitive level, onset of delirium, health related quality of life and functional capacity measures as primary endpoints, considering mandatory their repeated measure at 3,6 and 12 months and 5 years follow-up after a cardiovascular complex intervention.

This statement reinforces a key point of geriatric cardiology that considers patient-centered health status such as patients' symptoms relief, global functional capacity, quality of life and risk of disability as primary outcomes as well as all-cause or cardiovascular death. Unfortunately, we found that few studies used the aforementioned domains as primary outcome measures although so important from the elderly patient's point of view.

This lack of evidence was highlighted some years ago in an interesting review on BMJ [13] by Rahimi et al. that stressed the systematic underuse of patient reported outcomes in cardiovascular trials even after taking account of their relevance to individual trials. The authors underlined the need for selection of more patient focused outcomes and addressed the problems associated with composite outcomes and assessment of harms in cardiovascular trials. Nevertheless they invited researchers to design clinical trials selecting outcomes that are relevant and meaningful to those who potentially will be affected by them.

After complex cardiac surgery, a slow and complicated recovery course is present, especially in the elderly with high level of comorbidity; thus, preoperative and postoperative level of functional capacity play a crucial role to reach a good level of autonomy in the activities of daily living soon after surgery. In this perspective, AHA statement published recently in Circulation [14] points out the need to prioritizing global functional capacity as a vital goal of care for therapies oriented to older adults with cardiovascular disease.
A good degree of mental and physical autonomy after surgery is the key point to avoid disability for a long period of time. Therefore disability-free survival seems to satisfy the ideal characteristics of a patient-centered outcome after cardiac surgery (Figure 2). Nevertheless, the question is how and at which time we can quantify this parameter after cardiac surgery? Sure, the loss of one or more of activities of daily living according with Katz classification could be a valid and reliable instrument to measure this parameter. In addition, the discharge towards nursing home could be another surrogate outcome that could certify a significant loss of autonomy.

Finally, we know how the trajectory that leads from frailty to disability after surgery is not linear but it is often characterized by succession of improvement and worsening periods. This time-related phenomenon is mainly influenced by burden and severity of comorbidity, social and familial support, perioperative complications and preoperative global functional capacity; thus, it becomes mandatory repeating a measure of these domains in a follow-up period [9].

\section{Future perspectives}

Aiming to close in the next future these knowledge gaps in the elderly care, some choices are now urgent. First, we should develop new risk scores that include cognitive function, frailty, and measures of global functional capacity in the assessment of perioperative morbidity and mortality including disability free survival and quality of life as primary goals of care. Second, studies are needed to analyze the role of physical conditioning and pre-rehabilitation procedures before major cardiac surgery with the aim for reducing periprocedural risk and improving short and long term postprocedural outcomes.

Third, the collaborative work among cardiologists, anesthesiologist and geriatricians in the context of heart team must take into account the role of patients' centered outcomes before planning the decisionmaking process related to invasive and complex cardiovascular surgery procedures.

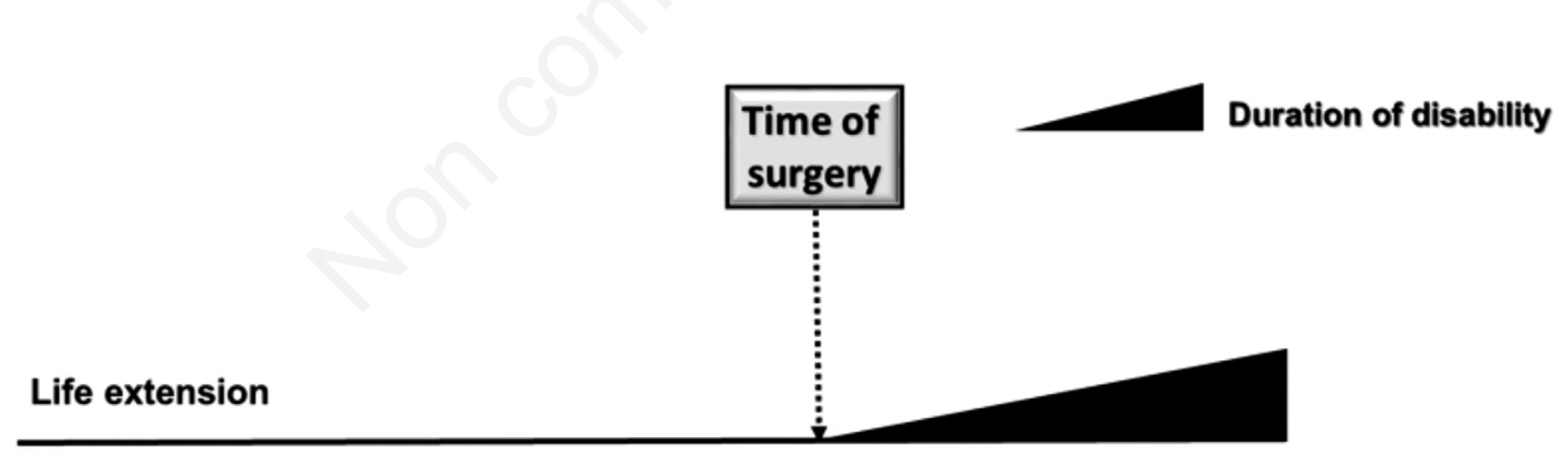

\section{Compression of Disability}

Disability free survival

Figure 2. Disability free-survival; a patient's centered primary outcome. 


\section{References}

1. Roger VL, Go AS, Lloyd-Jones DM, et al. Heart disease and stroke statistics - 2011 update: a report from the American Heart Association. Circulation 2011;123:e18-e209.

2. Buth KJ, Gainer RA, Legare JF, Hirsch GM. The changing face of cardiac surgery: Practice patterns and outcomes 2001-2010. Can J Cardiol 2014;30:224-30.

3. Wendt D, Thielmann M, Kahlert P et al. Comparison between different risk scoring algorithms on isolated conventional or transcatheter aortic valve replacement. Ann Thorac Surg 2014;97: 796-802.

4. Bergman H, Ferrucci L, Guralnik J, et al. Frailty: An emerging research and clinical paradigm - Issues and controversies. J Gerontol A Biol Sci Med Sci 2007;62:731-7.

5. Fried LP, Tangen CM, Walston J, et al. Cardiovascular Health Study Collaborative Research Group. Frailty in older adults: evidence for a phenotype. J Gerontol A Biol Sci Med Sci 2001;56:M146-56.

6. Rockwood K, Song X, MacKnight C, et al. A global clinical measure of fitness and frailty in elderly people. CMAJ 2005;173:489-95.

7. Afilalo J, Mottillo S, Eisenberg MJ et al. Addition of frailty and disability to cardiac surgery risk scores identifies elderly patients at high risk of mortality or major morbidity. Circ Cardiovasc Qual Outcomes 2012;5:222-8.
8. Afilalo J, Kim S, O'Brien S, et al. Gait speed and operative mortality in older adults following cardiac surgery. JAMA Cardiol 2016;1:314-21.

9. Freiheit EA, Hogan DB, Patten SB, et al. Frailty trajectories after treatment for coronary artery disease in older patients. Circ Cardiovasc Qual Outcomes 2016;9:230-8.

10. Spertus JA. Evolving applications for patient-centered health status measures. Circulation 2008;118:2103-110.

11. Rich MW, Chyun DA, Skolnick AH, et al. Knowledge gaps in cardiovascular care of the older adult population: a scientific statement from the American Heart Association, American College of Cardiology, and American Geriatrics Society. J Am Coll Cardiol 2016;67:2419-40.

12. Lansky AJ, Messé SR, Brickman AM, et al. Proposed standardized neurological endpoints for cardiovascular clinical trials: an academic research consortium initiative. J Am Coll Cardiol 2017;69: 679-91.

13. Rahimi K, Malhotra A, Banning AP, et al. Outcome selection and role of patient reported outcomes in contemporary cardiovascular trials: systematic review. BMJ 2010;341:1-8.

14. Forman DE, Arena R, Boxer R, et al. Prioritizing functional capacity as a principal end point for therapies oriented to older adults with cardiovascular disease. A scientific statement for healthcare professionals from the American Heart Association. Circulation 2017;135:e1-e25. 